PHYSICAL REVIEW D 89, 071902(R) (2014)

\title{
Direct detection of baryogenesis mechanism from squark decays at LHC
}

\author{
Haipeng $\mathrm{An}^{1}$ and Yue Zhang ${ }^{2}$ \\ ${ }^{1}$ Perimeter Institute, Waterloo, Ontario N2L 2Y5, Canada \\ ${ }^{2}$ California Institute of Technology, Pasadena, California 91125, USA
}

(Received 14 October 2013; published 9 April 2014)

\begin{abstract}
We investigate the feasibility of directly detecting a generation mechanism of the cosmic baryon asymmetry by repeating the same particle physics process inside the LHC. We propose a framework with $R$-parity and $C P$ violating squark decays responsible for baryogenesis, which can be embedded in supersymmetric models and is partly motivated by naturalness. We argue that the baryon number generation here is closely related to lepton charge asymmetry on the resonance. We emphasize the importance of the single charged lepton plus multijet channel in the absence of significant missing energy in search of such a scenario.
\end{abstract}

DOI: $10.1103 /$ PhysRevD.89.071902

PACS numbers: 11.30.Fs, 11.30.Er, 12.60.Jv, 13.85.Rm

\section{INTRODUCTION}

The existence of baryon asymmetry in our Universe is one of the mysteries and long-standing topics in particle physics and cosmology. Many theories have been proposed ever since to understand how the baryon asymmetry has been generated. One challenging issue to these theories is how to detect them today. There can be various indirect connections to other phenomena in low energy and collider experiments, from the $C P$ violations behind baryogenesis to the new states which lead to out-of-equilibrium conditions. While it seems difficult to reach the temperature for baryogenesis to happen in the current laboratories, the motivation of this work is to examine how the particle physics side of certain baryogenesis scenarios could be detected in a more direct way.

Natural supersymmetry (SUSY) was invented to solve the hierarchy problem, which necessarily contains light top squarks (stop) accessible to the LHC energy. The LHC data have already constrained the stop to be heavier than $\sim 700 \mathrm{GeV}$ [1] if it decays into neutralino, unless one resorts to a compressed spectrum. $R$-parity violation (RPV) is an alternative option to hide the light stops from the current LHC data [2]. The RPV interactions controlling the lifetime of the stop may also reach equilibrium and washout the primordial baryon number asymmetry in the early Universe [3]. Interestingly, the minimal value of such coupling to wash out the baryon number coincides with the one allowing a sub-TeV stop to decay promptly at colliders [4]. Therefore, the RPV interactions either wash out the primordial baryon asymmetry, or induce detectable displaced vertices in the LHC. In the first case, a new mechanism for late baryon number generation is needed.

In this paper, we propose a scenario for baryogenesis in which both the $C P$ and baryon number violations can be observed at colliders, and can be realized in the natural SUSY framework. The RPV decay of the lightest SUSY partner (LSP) is used to generate the baryon number. Two types of RPV interactions are needed, which break both baryon and lepton symmetries explicitly. The LSP in charge of the genesis can be stop itself or another lighter squark, and it had better be lighter than a few hundred GeV for the sake of naturalness. This allows the LSP squark to be copiously produced at the $14 \mathrm{TeV}$ LHC. We show that successful baryogenesis requires large $C P$ violation, which can be manifested at colliders via the lepton charge asymmetry in the decays of the squark resonances, and can serve as a smoking-gun signature of this scenario.

\section{TOY MODEL}

To capture the essence, we start with a toy model with two squarks $(i=1,2)$,

$$
\mathcal{L}=\lambda_{i}^{\prime \prime} \bar{b}^{c} P_{R} c \tilde{d}_{i}+\lambda_{i j}^{\prime}\left(\bar{u}_{j} P_{R} \mu^{c}-V_{j k} \bar{d}_{k} P_{R} \nu^{c}\right) \tilde{d}_{i},
$$

where $j, k=1,2,3$ and $V$ is the Cabbibo-KobayashiMaskawa (CKM) matrix. The above Lagrangian can be obtained from the minimal supersymmetric SM (MSSM) with RPV, $\tilde{d}_{i}$ identified as right-handed down-type squark, and all other superpartners decoupled. The quark flavors in the $\lambda^{\prime \prime}$ term and the muon flavor in $\lambda^{\prime}$ are chosen for illustration. To suppress the proton decay, we forbid the operators containing the first generation quarks explicitly, which implies $\lambda_{i 1}^{\prime}=0$ and $\lambda_{i 2}^{\prime} V_{21}+\lambda_{i 3}^{\prime} V_{31}=0$ [5]. The hierarchy of the CKM element predicts $d_{i}$ must couple preferably to the third generation quarks, and therefore (1) simplifies to

$$
\mathcal{L} \simeq \lambda_{i}^{\prime \prime} \bar{b}^{c} P_{R} c \tilde{d}_{i}+\lambda_{i}^{\prime}\left(\bar{t} P_{R} \mu^{c}-b P_{R} \nu^{c}\right) \tilde{d}_{i}
$$

The first thing to notice is that the existence of both $\lambda^{\prime}$ and $\lambda^{\prime \prime}$ type RPV interactions induces the proton decay [6]. With the above choice of flavors, the proton decay happens at two-loop order. We calculate its rate by using the chiral effective Lagrangian [7] and the lattice results on nucleonpion matrix elements [8]. The leading decay mode is $p \rightarrow K$, whose partial lifetime is constrained by Super-Kamiokande 
HAIPENG AN AND YUE ZHANG

[9], which is translated to the upper bound $\sqrt{\left|\lambda_{i}^{\prime \prime} \lambda_{i}^{\prime}\right|} \lesssim$ $2 \times 10^{-6}\left(m_{\tilde{d}_{i}} / 600 \mathrm{GeV}\right)^{2}$.

\section{BARYOGENESIS IN THE EARLY UNIVERSE}

We proceed to discuss how baryon asymmetry could be regenerated from the decays of $\tilde{d}_{i}$ via (2) [10]. The mechanism discussed here relies on $R$-parity and $C P$ violating decays, which is similar to leptogenesis $[11,12]$, except that the decaying particles are colored and not self-conjugate, and the baryon number is created directly from their decays. From Eq. (2), the squarks have the decay channels

$$
\tilde{d}_{i} \rightarrow \bar{b} \bar{c}, \quad t \mu^{-}(b \nu), \quad \tilde{d}_{i}^{*} \rightarrow b c, \quad \bar{t} \mu^{+}(\bar{b} \bar{\nu}) .
$$

The $\tilde{d}_{i}$ decays generate only $B+L$, which is the difference of quantum numbers between the two final states [13]. In order for baryon number to survive, the decay must happen after the weak sphaleron process ceases.

We define the $C P$ violation in $\tilde{d}_{i}$ and $\tilde{d}_{i}^{*}$ decays and a hadronic branching ratio $\mathrm{Br}_{\tilde{d}_{i} \rightarrow \bar{b} \bar{c}}$ as follows

$\varepsilon_{i} \equiv \frac{\Gamma_{\tilde{d}_{i} \rightarrow \bar{b} \bar{c}}-\Gamma_{\tilde{d}_{i}^{*} \rightarrow b c}}{\Gamma_{\tilde{d}_{i} \rightarrow \bar{b} \bar{c}}+\Gamma_{\tilde{d}_{i}^{*} \rightarrow b c}}, \quad \operatorname{Br}_{i} \equiv \frac{\Gamma_{\tilde{d}_{i} \rightarrow \bar{b} \bar{c}}}{\Gamma_{\tilde{d}_{i} \rightarrow \bar{b} \bar{c}}+2 \Gamma_{\tilde{d}_{i} \rightarrow t \mu^{-}}}$

All the other decay branching ratios can be obtained from these two quantities,

$$
\begin{aligned}
\operatorname{Br}_{\tilde{d}_{i} \rightarrow t \mu^{-}} & =\mathrm{Br}_{\tilde{d}_{i \rightarrow b \nu}}=\frac{1}{2}\left(1-\mathrm{Br}_{i}\right), \quad \mathrm{Br}_{\tilde{d}_{i}^{*} \rightarrow b c}=\frac{1-\varepsilon_{i}}{1+\varepsilon_{i}} \mathrm{Br}_{i}, \\
\operatorname{Br}_{\tilde{d}_{i}^{*} \rightarrow \bar{t} \mu^{+}} & =\mathrm{Br}_{\tilde{d}_{i}^{*} \rightarrow \bar{b} \bar{\nu}}=\frac{1}{2}\left(1-\frac{1-\varepsilon_{i}}{1+\varepsilon_{i}} \mathrm{Br}_{i}\right)
\end{aligned}
$$

To generate the correct sign of baryon asymmetry requires $\varepsilon_{i}<0$. The source term of the Boltzmann equation depends on the quantity

$$
\varepsilon_{i} \mathrm{Br}_{i}=\frac{\operatorname{Im}\left(\lambda_{i}^{\prime \prime} \lambda_{i}^{\prime} \lambda_{j}^{\prime \prime *} \lambda_{j}^{*}\right)}{\left(\left|\lambda_{i}^{\prime \prime}\right|^{2}+\left|\lambda_{i}^{\prime}\right|^{2}\right)\left(\left|\lambda_{j}^{\prime \prime}\right|^{2}+\left|\lambda_{j}^{\prime}\right|^{2}\right)} F_{j}\left(m_{j}^{2} / m_{i}^{2}\right),
$$

where $\quad F_{j}(x)=\left(2 \Gamma_{j} / m_{j}\right)[1 /(1-x)-3+(2+3 x) \underset{\sim}{ } \times$ $\ln (1+1 / x)]$ and $m_{i}, \Gamma_{i}$ are the mass and width of $\tilde{d}_{i}$, respectively. When $\tilde{d}_{1}$ and $\tilde{d}_{2}$ become quasidegenerate, the resonant propagator is regularized by $\Gamma_{2}$ [14], $F_{j}(x) \approx$ $\left(m_{1}-m_{2}\right)\left(\Gamma_{2} / 2\right) /\left[\left(m_{1}-m_{2}\right)^{2}+\left(\Gamma_{2} / 2\right)^{2}\right]$.

The Boltzmann equation to generate the baryon number is

$\frac{d Y_{B}}{d z}=-\frac{2 \varepsilon_{i} \Gamma_{i}^{\prime \prime}}{H z}\left(Y_{\tilde{d}_{i}}-Y_{\tilde{d}_{i}}^{\mathrm{eq}}\right)-\frac{\left(4 \Gamma_{i}^{\prime \prime}+\Gamma_{i}^{\prime}\right) Y_{\tilde{d}_{i}}^{\mathrm{eq}}}{H(z) z} \frac{Y_{B}}{Y_{q}^{\mathrm{eq}}}+\cdots$

where $z=M_{\tilde{d}_{1}} / T, Y_{i} \equiv n_{i} / s$ is the yield of $\tilde{d}_{i}, s=$ $2 \pi^{2} g_{* S} T^{3} / 45$ is the total entropy density. The term
PHYSICAL REVIEW D 89, 071902(R) (2014)

proportional to $\varepsilon_{i}$ is the source term, which implies $\tilde{d}_{i}$ must decay out of equilibrium. In the washout terms, we have defined $\Gamma_{i}^{\prime} \equiv 2\left\langle\Gamma_{i}\right\rangle\left(\mathrm{Br}_{\tilde{d}_{i} \rightarrow t \mu^{-}}+\mathrm{Br}_{\tilde{d}_{i}^{*} \rightarrow \bar{t} \mu^{+}}\right)$and $\Gamma_{i}^{\prime \prime} \equiv$ $\left\langle\Gamma_{i}\right\rangle\left(\mathrm{Br}_{\tilde{d}_{i} \rightarrow \bar{b} \bar{c}}+\mathrm{Br}_{\tilde{d}_{i}^{*} \rightarrow b c}\right)$, where \langle\rangle means thermal average. The $\cdots$ are the washout terms involving the lepton asymmetry and are numerically insignificant.

Neglecting the asymmetry between the numbers of $\tilde{d}_{i}$ and $\tilde{d}_{i}^{*}$, which is already exponentially suppressed during the decay, the Boltzmann equation governing the $\tilde{d}_{i}$ number density can be written as

$\frac{d Y_{\tilde{d}_{i}}}{d z}=-\frac{\left\langle\Gamma_{i}\right\rangle}{H(z) z}\left(Y_{\tilde{d}_{i}}-Y_{\tilde{d}_{i}}^{\mathrm{eq}}\right)-\frac{s\left\langle\sigma v_{i}\right\rangle}{H(z) z}\left(Y_{\tilde{d}_{i}}^{2}-\left(Y_{\tilde{d}_{i}}^{\mathrm{eq}}\right)^{2}\right)$,

where $\sigma v_{i}$ include all possible $\tilde{d}_{i} \tilde{d}_{i}^{*} \rightarrow \bar{q} q, g g$ annihilation channels. The strong interaction keeps $\tilde{d}_{i}$ in equilibrium and tends to suppress $Y_{\tilde{d}_{i}}-Y_{\tilde{d}_{i}}^{\mathrm{eq}}$, and consequently the final baryon asymmetry. If the decay mainly happens well after the freeze-out time $z^{\mathrm{fo}}$, defined by $n_{\tilde{d}_{i}}^{\mathrm{eq}}\langle\sigma v\rangle\left(z^{\mathrm{fo}}\right) \sim H\left(z^{\mathrm{fo}}\right)$, the resulting $Y_{B}(\infty) \sim \varepsilon Y_{\tilde{d}_{i}}^{\mathrm{eq}}\left(z^{\mathrm{fo}}\right)$ will be much smaller than the observed value.

On the other hand, if the decay happens during the freeze-out, the final baryon number can be enhanced [15]. Combining Eqs. (7) and (8) and neglecting the washout terms, $d Y_{B} / d z \sim \varepsilon_{i} \mathrm{Br}_{i}\left\langle\Gamma_{i}\right\rangle /\left(\left\langle\Gamma_{i}\right\rangle+2 n_{\tilde{d}_{i}}^{\mathrm{eq}}\left\langle\sigma v_{i}\right\rangle\right)\left(d Y_{\tilde{d}_{i}}^{\mathrm{eq}} / d z\right)$. For $z \ll 1$, the annihilation rate dominates over the decay, whereas at $z \gg z^{\mathrm{fo}}, d Y_{\tilde{d}_{j}}^{\mathrm{eq}} / d z$ is Boltzmann suppressed. Therefore, the dominant contribution to $Y_{B}$ is from the epoch $z \sim z^{\mathrm{eq}}$, with $n_{\tilde{d}_{i}}^{\mathrm{eq}}\left\langle\sigma v_{i}\right\rangle \sim\left\langle\Gamma_{\tilde{d}_{i}}\right\rangle$. The resulting baryon number is $Y_{B}(\infty) \sim \varepsilon_{i} Y_{\tilde{d}_{i}}^{\mathrm{eq}}\left(z^{\mathrm{eq}}\right)$. Thus, it can be enhanced by orders of magnitude if $z^{\mathrm{eq}} \ll z^{\text {fo }}$. As a result, for a given $\varepsilon$, the final baryon asymmetry first increases with the decay rate (Fig. 1), and then drops at larger decay rate because the washout terms in (7) become important. From Fig. 1, we

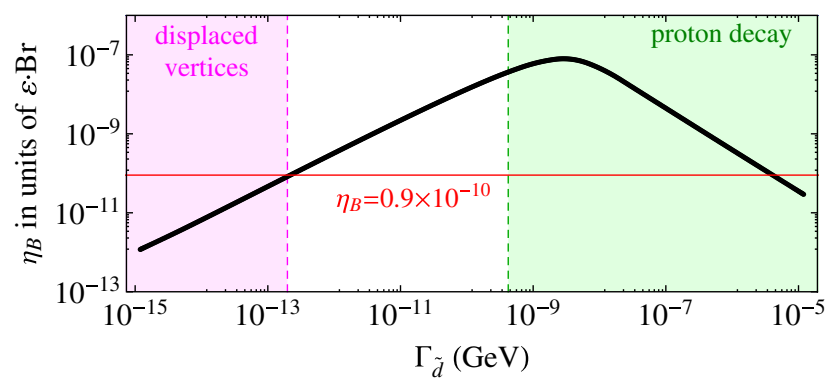

FIG. 1 (color online). Thick solid curve represents final baryon asymmetry in units of $\varepsilon \mathrm{Br}$ as a function of sdown total width, for $M_{\tilde{d}}=600 \mathrm{GeV}$. The suppression in the asymmetry at large decay width is due to the stronger washout effects. The horizontal line is the observed baryon asymmetry. The shaded region on the right is excluded by proton decay, and in the shaded region on the left, sdown decay is displaced at LHC. 
DIRECT DETECTION OF BARYOGENESIS MECHANISM ...

also find that successful baryogenesis requires $\varepsilon \gtrsim 10^{-2}$, which implies the mass gap between $\tilde{d}_{1}$ and $\tilde{d}_{2}$ should be at most $10^{2}$ of their widths.

\section{BARYOGENESIS INSIDE THE LHC}

As colored particles, $\tilde{d}_{i} / \tilde{d}_{i}^{*}$ can be copiously pairproduced at high-energy colliders. This offers a unique opportunity to access the particle physics part of the above baryogenesis scenario.

\section{A. Constraints}

After being produced inside the LHC, $\tilde{d}_{i}$ and $\tilde{d}_{i}^{*}$ will decay according to (3). The possible final states are

\begin{tabular}{lcc}
\hline Process & Signal & Relevant data \\
\hline$(\bar{b} \bar{c})(b c)$ & $4 j$ & $\ldots$ \\
& $\mu^{+} \mu^{-} 2 b 4 j$ & Leptoquark [16] \\
$\left(t \mu^{-}\right)\left(\bar{t} \mu^{+}\right)$ & $\mu^{+} \mu^{-} \ell^{ \pm} 2 b 2 j E_{T}$ & Chargino- \\
& $\mu^{+} \mu^{-} \ell^{+} \ell^{\prime} 2 b E_{T}$ & Neutralino [17] \\
& $\mu^{ \pm} 2 b 2 j E_{T}$ & Leptoquark [16] \\
$\left(t \mu^{-}\right)(\bar{b} \bar{\nu}),\left(\bar{t} \mu^{+}\right)(b \nu)$ & $\mu^{ \pm} \ell^{\mp} 2 b E_{T}$ & Stop [18] \\
$(b \nu)(\bar{b} \bar{\nu})$, & $2 b E_{T}$ & Sbottom [19] \\
$(b \nu)(b c),(\bar{b} \bar{\nu})(\bar{b} \bar{c})$ & $2 b 1 j E_{T}$ & Multijet + $E_{T}[20]$ \\
$\left(t \mu^{-}\right)(b c),\left(\bar{t} \mu^{+}\right)(\bar{b} \bar{c})$ & $\mu^{ \pm} 2 b 3 j$ & Our signal \\
& $\mu^{ \pm} \ell^{\mp} 2 b 1 j E_{T}$ & \\
\hline
\end{tabular}

with $\ell, \ell^{\prime}=e, \mu$, and their branching ratios can be calculated from Eq. (5). The corresponding LHC data relevant to the constraints are also shown. There is no constraint from the $4 j$ channel, which is the usual place to hide SUSY using RPV. We find that the $\mu^{+} \mu^{-}+$jets and $\mu^{ \pm}+$jets $+E_{T}$ channels can be constrained by simple leptoquark searches [16], which give the strongest limits. They have been interpreted to the bounds on $\mathrm{Br}$ and $\varepsilon$, and displayed in Fig. 2.

The searches for pair-produced dijet resonances in the four-jet final states at the LHC may also be sensitive to this model, but the constraints from current searches are not sensitive to the RPV SUSY models [21].

\section{B. Resonances and lepton charge asymmetry}

Compared to other channels the analysis of $\mu^{+}$jets and $\mu^{-}$jets without significant missing energy has received less motivations. However, these channels can be used as a smoking-gun signal for the baryogenesis scenario discussed above, since $\tilde{\sim} \tilde{d}$ decays preferably to semileptonic channels, whereas $\tilde{d}^{*}$ to hadronic channels. Therefore, if the events are triggered with a single muon and multiple hard jets, it is expected to see more $\mu^{-}$events than $\mu^{+}$ events. In practice, we use PYTHIA 8 [22] and FASTJET 3 [23] to generate the decay events of the pair-produced $\tilde{d}$ and $\tilde{d}^{*}$. We require the transverse momenta $\left(P_{T}\right)$ of the $\mu^{ \pm}$to be larger than $170 \mathrm{GeV}$. The two hardest jets are required to
PHYSICAL REVIEW D 89, 071902(R) (2014)

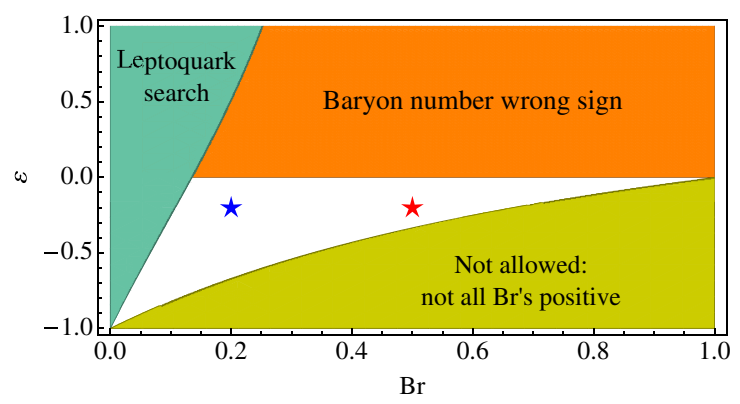

FIG. 2 (color online). Constraints on the $\mathrm{Br}-\varepsilon$ parameter space. The squark mass is taken equal to $600 \mathrm{GeV}$. The shaded region in the upper-left corner is excluded by the leptoquark data. The shaded region in the upper-right corner is excluded for generating a wrong sign of baryon asymmetry. The shaded region in the lower-right corner is theoretically not accessible. We note the leptoquark bound shrinks for heavier squark, and will disappear for the $800 \mathrm{GeV}$ case. The stars represent the sample values of $\mathrm{Br}$ and $\varepsilon$ used in the Monte Carlo simulation.

have $P_{T}>200 \mathrm{GeV}$, and the third hardest jet $P_{T}>150 \mathrm{GeV}$. We calculate the invariant mass of two of the jets $M_{j j}$, and compare it with the invariant mass of the muon and the rest of the jets $M_{\mu j}$. By finding the combination with the closest $M_{j j}$ and $M_{\mu j}$, we identify the mass of $\tilde{d}$ with $M_{j j}$. To reduce the background from $W+$ jets, we further require the missing energy to be smaller than $30 \mathrm{GeV}$. With these cuts, the major background comes from QCD multijet processes with one jet misidentified as an muon. For $P_{T}>100 \mathrm{GeV}$, the fake rate is less than $10^{-4}$ [24]. To be conservative, we take the fake rate equal to this upper limit.

The $M_{j j}$ distributions for events with a single $\mu^{ \pm}$for $m_{\tilde{d}}=600$ and $800 \mathrm{GeV}$ are shown in Fig. 3, where we choose two benchmark points corresponding to the blue and red stars shown in Fig. 2. For the first benchmark, with $\varepsilon=-0.2$ and $\mathrm{Br}=0.2$, the ratio of the parton level
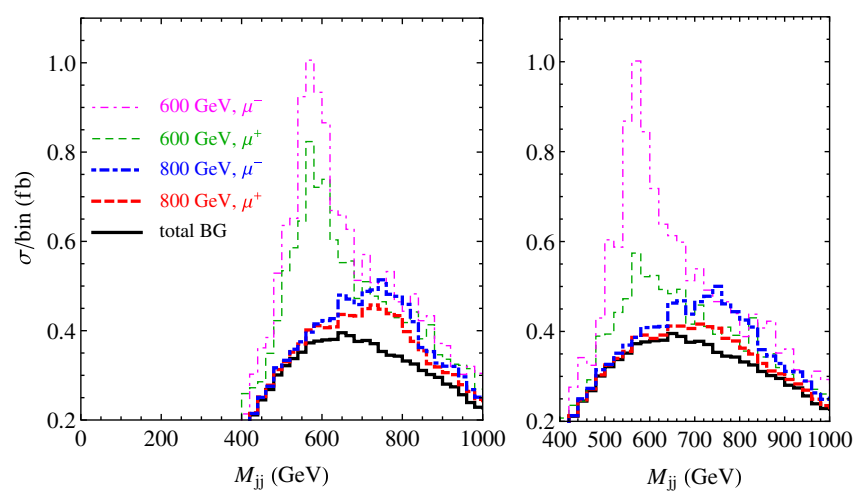

FIG. 3 (color online). Invariant mass distribution for dijet $M_{j j}$ (selected equal to $M_{\mu^{ \pm} t}$, see text) in LHC with $14 \mathrm{TeV}$ center-ofmass energy, $m_{\tilde{d}}=600$ and $800 \mathrm{GeV}$, and $\varepsilon=-0.2, \mathrm{Br}=0.2$ (left) and $\varepsilon=-0.2, \mathrm{Br}=0.5$ (right). The thick curve shows the total background assuming the muon fake rate to be $10^{-4}$. Signal and background are stacked together. 
production rates of the $\mu^{-}$jets and $\mu^{+}$jets events can be calculated from Eq. (5) that $\hat{\sigma}_{\mu^{-} \text {jets }} / \hat{\sigma}_{\mu^{+} \text {jets }} \approx 1.7$. For the second benchmark, we have $\hat{\sigma}_{\mu^{-} \text {jets }} / \hat{\sigma}_{\mu^{+} \text {jets }}=3$. In practice, the ratios of heights of the $\mu^{-}$peak to $\mu^{+}$peak shown in Fig. 3 are smaller than their parton level values, due to the self-contaminations from other decay channels of the resonances, apart from the SM background. One such contamination is from the $4 j$ channel, which is potentially more important, for it has the same bump structure as the desired $\mu^{ \pm}$jets signal.

\section{Identification of baryogenesis at the LHC}

From the above discussion, we are able to observe a particle or its antiparticle (on resonance) decaying into one muon and one top quark, or two hard jets. Based on the fact that a hard jet can be either a quark/antiquark or a gluon (we assume that jet substructure analysis can distinguish jets from boosted heavy particles), we enumerate all the possibilities of the color and spin quantum numbers, which can be reconstructed from the two types of final states, using $\mu(1,1 / 2), \quad t(3,1 / 2), \quad j=g(8,1), \quad q(3,1 / 2) \quad$ or $\bar{q}(\overline{3}, 1 / 2)$. The only choices for the quantum numbers of $(\mu t)$ and $(j j)$ to match are color triplets with integer spins. Meanwhile, the dijet final states decayed from the resonances are also fixed to be $\left(\bar{q} \bar{q}^{\prime}\right)$ and $\left(q q^{\prime}\right)$. We further assume that the resonance is made of particle-antiparticle pairs $(X$ and $\bar{X})$. Then, from the lepton charge asymmetry between $\left(\mu^{-} t\right)$ and $\left(\mu^{+} \bar{t}\right)$, we know the semileptonic decay branching ratios of $X$ and $\bar{X}$ are different, and so are the hadronic decays due to the $C P T$ theorem which dictates that the equality of the total widths of $X$ and $\bar{X}$. Therefore, a net baryon number must be generated if they are pair produced. Another caveat to this argument is the possibility of same sign pair productions of $X X$ and $\bar{X} \bar{X}$. Because LHC is a proton-proton machine, the asymmetry of the light quark parton distributions results in the different production rates of $X X, \bar{X} \bar{X}$, with the exchange of a $t$-channel gluino, if $X$ is the superpartner of a light quark. However, these processes are always accompanied by events with same-sign di-muon plus jets. The identification of baryogenesis at LHC requires the absence of this kind of events. They could be suppressed with a gluino mass larger than $5 \mathrm{TeV}$.

\section{A COMPLETE MODEL}

To generate enough baryon asymmetry, the mass gap between $\tilde{d}_{1}$ and $\tilde{d}_{2}$ should be of the same order as their decay widths, which is suppressed by their RPV couplings (see Fig. 1), $\sim 10^{-12} \mathrm{GeV}$. In a natural model, such a small mass gap should be controlled by the same RPV couplings as well, which implies the existence of an approximate $S U(2)_{h}$ horizontal symmetry. Here we give a SUSY model example to illustrate the idea. For the sake of the gauge unification, we add two pairs of $\mathbf{5}_{i}^{\prime}$ and $\overline{\mathbf{5}}_{i}^{\prime}$ to the MSSM, with $i=1,2$ forming doublets under the $S U(2)_{h}$ symmetry. We assume the $S U(2)_{h}$ symmetry is broken only by the RPV interactions in the superpotential $W=$ $W_{\mathrm{MSSM}}^{\mathrm{RPV}}+\lambda_{i}{ }^{\prime} Q_{2} L_{1} D_{i}^{\prime c}+\lambda_{i}^{\prime \prime} U_{2}^{c} D_{3}^{c} D_{i}^{\prime c}+M D_{i}^{\prime} D_{i}^{\prime c}$, where $D_{i}^{\prime c} \in \overline{\mathbf{5}}_{i}^{\prime}$, and the above $\tilde{d}_{i}^{*}$ in the sdown case can be identified as the scalar part of $D_{i}^{\prime c}=\tilde{d}_{i}^{*}+\theta d_{i}^{\prime}$. The loop generated mass difference between $\tilde{d}_{1}$ and $\tilde{d}_{2}$ is just on the same order of magnitude as their widths. The masses of $\tilde{d}_{i}^{*}$ and its fermionic partner $d_{i}^{\prime}$ can be different due to a SUSY breaking but $S U(2)_{h}$ conserving soft mass. It is possible to arrange $d_{i}^{\prime}$ to decay into a quark and a squark via the same RPV interactions. We find the gauge couplings are still perturbative at the unification scale.

\section{REALIZATION IN THE MSSM}

A more interesting question is how to realize the toy model in the MSSM. Again, we need a tiny mass gap between two lightest squarks, otherwise the $C P$ violation parameter is linearly suppressed by the mass difference. This degeneracy suffers from quantum corrections from different Yukawa couplings for different flavors, and requires tuning of order $10^{-5}$ (with choice of squark flavors below). The real problem is that, in the early Universe, the finite temperature effect modifies the mass gap dynamically. The most important contribution comes from the F-terms of the Yukawa couplings, with the Higgs boson running in the thermal loop,

$\left(m_{1}-m_{2}\right)(T) \approx \Delta m_{0}+\frac{y_{1}^{2}-y_{2}^{2}}{2 m_{\tilde{q}_{1}} M_{h}}\left(\frac{M_{h} T}{2 \pi}\right)^{3 / 2} e^{-M_{h} / T}$,

where low temperature expansion [25] has been used, and $M_{h}=126 \mathrm{GeV}$. Through Eq. (6), this causes the $C P$ violation parameter $\varepsilon$ to vary with the temperature. Enough baryon asymmetry requires the source term for baryogenesis to remain effective, i.e., $\varepsilon(T)>10^{-2}$, for a long enough period around the freeze-out temperature $T_{f} \sim 20 \mathrm{GeV}$. As a result, there is an upper bound on the differences of Yukawa couplings $\left|y_{1}^{2}-y_{2}^{2}\right|<10^{-5}$. This limits the choice of flavors to the nearly-degenerate sdownsstrange co-LSP scenario only, which is able to give sufficient baryon asymmetry. In this case, for naturalness, the light stop can still be hidden by either cascade decaying to the LSPs or via its own RPV couplings.

\section{SUMMARY}

To summarize, we propose a $\mathrm{TeV}$ scale baryogenesis picture, which can be realized in natural SUSY models, and can be directly probed at the LHC. The baryogenesis from the decay of colored particle requires $C P$ violation to be close to maximal, which can be realized with nearly degenerate squarks. The smoking-gun signal would be the charge asymmetry from the decay of squark resonances. 
The charged lepton plus multijet final states without significant missing energy, should be taken into account in future analysis of LHC data, which may unveil the origin of baryon asymmetry of our Universe.

\section{Acknowledgement}

We acknowledge insightful discussions with Clifford Cheung, Tao Liu, Michael Ramsey-Musolf, Goran
Senjanović and Natalia Toro. H. A.'s research at Perimeter Institute is supported by the Government of Canada through Industry Canada and by the Province of Ontario through the Ministry of Research and Innovation. Y.Z.'s work is supported by the Gordon and Betty Moore Foundation through Grant No. 776 to the Caltech Moore Center for Theoretical Cosmology and Physics, and by the D.O.E. Grant No. DE-FG02-92ER40701.
[1] G. Aad et al. (ATLAS Collaboration), Phys. Rev. Lett. 109, 211802 (2012); 109, 211803 (2012); 11 (2012) 094; CMS Collaboration, Report No. CMS PAS SUS-13-004; S. Chatrchyan et al. (CMS Collaboration), Eur. Phys. J. C 73, 2677 (2013).

[2] C. Brust, A. Katz, S. Lawrence, and R. Sundrum, J. High Energy Phys. 03 (2012) 103; C. Csaki, Y. Grossman, and B. Heidenreich, Phys. Rev. D 85, 095009 (2012); J. A. Evans and Y. Kats, J. High Energy Phys. 04 (2013) 028; Y. Cui and R. Sundrum, Phys. Rev. D 87, 116013 (2013); R. Franceschini and R. Torre, Eur. Phys. J. C 73, 2422 (2013); R. Franceschini and R. N. Mohapatra, J. High Energy Phys. 04 (2013) 098; G. Krnjaic and Y. Tsai, arXiv:1304.7004; B. Batell, T. Lin, and L. -T. Wang, J. High Energy Phys. 01 (2014) 075.L. Di Luzio, M. Nardecchia, and A. Romanino, Phys. Rev. D 88, 115008 (2013);

[3] R. Barbier, C. Berat, M. Besancon, M. Chemtob, A. Deandrea, E. Dudas, P. Fayet, and S. Lavignac et al., Phys. Rep. 420, 1 (2005).

[4] P. W. Graham, D. E. Kaplan, S. Rajendran, and P. Saraswat, J. High Energy Phys. 07 (2012) 149.

[5] Based on the structures of renormalization group running of the RPV couplings, see, e.g., B. C. Allanach, A. Dedes, and H. K. Dreiner, Phys. Rev. D 60, 056002 (1999); 86039906 (E) (2012), we find it only requires a few percent of tuning for these relations to hold.

[6] A. Y. Smirnov and F. Vissani, Phys. Lett. B 380, 317 (1996).

[7] M. Claudson, M. B. Wise, and L. J. Hall, Nucl. Phys. B195, 297 (1982).

[8] S. Aoki et al. (JLQCD Collaboration), Phys. Rev. D 62, 014506 (2000); Y. Aoki, P. Boyle, P. Cooney, L. Del Debbio, R. Kenway, C. Maynard, A. Soni, and R. Tweedie (RBCUKQCD Collaboration), Phys. Rev. D 78, 054505 (2008).

[9] K. Kobayashi et al. (Super-Kamiokande Collaboration), Phys. Rev. D 72, 052007 (2005).

[10] For earlier studies on late time baryogenesis from gravitino decay, see, e.g., S. Dimopoulos and L. J. Hall, Phys. Lett. B
196, 135 (1987); J. M. Cline and S. Raby, Phys. Rev. D 43, 1781 (1991); K. Kohri, A. Mazumdar, and N. Sahu, Phys. Rev. D 80, 103504 (2009).

[11] M. Fukugita and T. Yanagida, Phys. Lett. B 174, 45 (1986).

[12] Earlier discussion about the possibility of detecting $\mathrm{TeV}$ resonant leptogenesis at LHC can be found in S. Blanchet, Z. Chacko, S. S. Granor, and R. N. Mohapatra, Phys. Rev. D 82, 076008 (2010).

[13] D. V. Nanopoulos and S. Weinberg, Phys. Rev. D 20, 2484 (1979).

[14] A. Pilaftsis and T. E. J. Underwood, Nucl. Phys. B692, 303 (2004).

[15] T. Hambye, New J. Phys. 14, 125014 (2012).

[16] The CMS Collaboration, Report No. CMS PAS EXO-12042.

[17] The ATLAS Collaboration, Report No. ATLAS-CONF2013-035.

[18] The ATLAS Collaboration, Report No. ATLAS-CONF2013-065.

[19] G. Aad et al. (ATLAS Collaboration), J. High Energy Phys. 10 (2013) 189.

[20] The CMS Collaboration, Report No. CMS PAS SUS-13012; The ATLAS Collaboration, Report No. ATLASCONF-2013-047.

[21] S. Chatrchyan et al. (CMS Collaboration), Phys. Rev. Lett. 110, 141802 (2013); T. Aaltonen et al. (CDF Collaboration), Phys. Rev. Lett. 111, 031802 (2013).

[22] T. Sjöstrand, S. Mrenna, and P. Skands, J. High Energy Phys. 05 (2006) 026; Comput. Phys. Commun. 178, 852 (2008).

[23] M. Cacciari, G. P. Salam, and G. Soyez, Eur. Phys. J. C 72, 1896 (2012); M. Cacciari and G. P. Salam, Phys. Lett. B 641, 57 (2006).

[24] A. J. Barr et al. (ATLAS Collaboration), Report No. ATL-PHYS-PUB-2009-068.

[25] G. W. Anderson and L. J. Hall, Phys. Rev. D 45, 2685 (1992). 